\title{
Estudo sobre as diferenças de percepção dos fatores relacionais entre empresa e terceirizado
}

\author{
Cleverson Regis Lino Marchalek \\ Marcelo Giroto Rebelato \\ PUCPR \\ Isabel Cristina Rodrigues \\ Faculdade de Administração e Negócios de Monte Alto
}

\begin{abstract}
Resumo
Este trabalho tem como objetivo o estudo das diferenças de percepção dos fatores relacionais no relacionamento entre contratante e terceirizado considerando-se o ambiente onde há múltiplos terceirizados competindo para o fornecimento a uma única empresa contratante. Ele se propõe a auxiliar empresas contratantes e empresas terceirizadas na construção de um relacionamento de longo prazo. Para tanto, o estudo apresenta um embasamento teórico focado nos modelos de relacionamento interorganizacionais, além de um estudo de casos múltiplos para identificação dos fatores críticos de sucesso, tanto do ponto de vista do contratante quanto do ponto de vista do fornecedor. Ao final, conclui-se que os fatores comunicação, confiança e divisão de riscos/recompensas são os que apresentam maior discrepância de percepção entre as empresas contratantes e terceirizadas.
\end{abstract}

\section{A study about the differences in the perception of relationship factors between contractors and outsourced}

\begin{abstract}
The objective of this work is to study the differences in the perception of the different levels of relationship between contractors and suppliers bearing in mind an environment wherein there are several suppliers competing to deliver service to one contractor. It aims to help contractors and suppliers to build up a long-term relationship. The study focuses on the existing models of inter-organizational relationships, as well as the analysis of multiple cases, so as to identify critical factors that lead to success, both from the point of view of the contractor and the supplier. The conclusion it arrives at is that the biggest discrepancy factors in terms of mutual perception between contractors and suppliers are: communication, trust and the sharing of risks and rewards.
\end{abstract}

Key words

Relationship between contractor and suppliers, perception gaps and outsourcing. 


\section{INTRODUĈ̣̃O}

Terceirizar parte dos processos de produção interna a fornecedores externos constitui-se em uma opção estratégica viável para as empresas que buscam obter vantagem competitiva através do foco em suas competências essenciais (PRAHALAD; HAMEL, 1990). Para a empresa contratante, uma terceirização bemsucedida pode trazer benefícios tais como a contenção e redução de custos, o acesso a inovações, a expansão da oferta de serviços, além da possibilidade de focar-se apenas nas atividades centrais (PRADO; TAKAYOKA, 2001; OLIVEIRA; SANTOS, 2000; ALMEIDA; SILVA, 1999; REZENDE, 1997; LEITE, 1994; BRASIL, 1993).

Entretanto, o relacionamento entre a empresa contratante e a empresa contratada pode ser tão complexo quanto o relacionamento entre dois seres humanos (ZINELDIN et al., 1997). Neste ambiente, comportamentos oportunistas não são raros. Por exemplo, um terceirizado pode aumentar o seu preço num momento de escassez do produto/serviço, ou um cliente pode encorajar a empresa contratada a garantias e benefícios extras, mesmo pretendendo substituí-la em um futuro próximo. Além disso, de acordo com Faria (1994), no Brasil é comum o estabelecimento de um modelo de terceirização baseado apenas na busca por redução de custos. Nestes casos, verifica-se uma grande dispersão de expectativas mútuas entre a empresa contratante e a empresa terceirizada. Ambas enxergam sua interação através de lentes distintas e o relacionamento interorganizacional fica distante da parceria e do comprometimento mútuo. Para Rezende (1997), o gerenciamento do relacionamento com os fornecedores é uma das principais dificuldades para o sucesso das terceirizações no Brasil.

Dessa forma, as diferenças de percepção entre o ponto de vista da empresa e do terceirizado prejudicam a cooperação, o comprometimento e a parceria interorganizacional. Os níveis de importância atribuídos a cada "fator crítico de sucesso" (FCS) (WATSON et al., 1982), por parte do contratante e do terceirizado, podem não ser coincidentes. Estas diferenças de percepção podem arruinar o processo de terceirização (MARCHALEK, 2005).

Desse modo, o objetivo deste artigo é identificar, por meio da pesquisa bibliográfica, e analisar, por meio de uma pesquisa de campo, as diferenças de percepção dos fatores relacionais no relacionamento entre contratante e fornecedor no contexto de diversos terceirizado fornecendo produtos/ serviços a uma única empresa contratante.

Dentro desse contexto, a pesquisa de campo foi realizada em empresas nacionais dos setores metalmecânico, financeiro e petroquímico. $\mathrm{O}$ estudo mostra-se relevante na medida em que oferece subsídios para uma melhor compreensão do fenômeno do relacionamento interorganizacional, auxiliando no entendimento mútuo entre as empresas e no estabelecimento de uma verdadeira relação de parceria baseada em expectativas conhecidas e reciprocamente esclarecidas.

\section{relacionamento entre a empresa contratante e a empresa contratada ser tão complexo quanto o relacionamento entre dois seres humanos.}

\section{O RELACIONAMENTO INTEREMPRESAS}

A situação econômica atual, marcada pela internacionalização dos mercados, crescimento da integração horizontal e vertical e crescimento do relacionamento entre empresas modificou a maneira pela qual a produção é organizada e a maneira pela qual as empresas se relacionam com o mercado. Os desafios encontrados reforçaram a importância do paradigma do relacionamento. Este paradigma é marcado pela mudança do relacionamento tradicional com os fornecedores para um relacionamento mais inovativo. Esta mudança seguiu uma linha evolucionária em duas fases: uma fase inicial de reforço do relacionamento nos aspectos logístico-produtivos (de 1970 a 1980) e uma segunda fase (1990 até hoje) em que há um relacionamento integrado até níveis estratégicos, algumas vezes classificados como parcerias. A passagem da relação tradicional para uma relação logística e mais recentemente para uma relação de parceria fez com que o grau de integração e cooperação entre compradores e fornecedor crescesse continuamente. Contribuíram para estas mudanças a ascensão de inovações técnicas e de gerenciamento (Just-in-Time, Total Quality Management, Advanced Manufacturing Technologies) e as pressões por constantes inovações de produtos e desenvolvimento tecnológico (VILLA; PANIZZOLO, 1996; ZINELDIN; JONSSON, 2000).

O relacionamento entre comprador e fornecedor está sob uma profunda evolução, cuja principal característica é a mudança da lógica do produto para a lógica da integração/ cooperação (RING; VAN de VEM, 1992; VILLA; PANIZZOLO, 1996; MCIVOR et al., 1998). A troca entre fornecedor-comprador não está mais simplesmente relacionada à troca física de objetos, mas também está relacionada a uma série de outros serviços, como por exemplo P\&D, design, informação, logística etc.

O conceito de aproximação nos relacionamentos comerciais, pregado pelo marketing de relacionamento, pode ser 
aplicado para os relacionamentos entre compradores e fornecedores. Para Izquierdo e Cillán (2004), o mercado industrial é melhor caracterizado por relacionamentos de longo prazo, estáveis e interativos, do que pela mudança. Novas práticas de gerenciamento emergiram baseadas na manutenção de relacionamentos estratégicos com um número limitado de fornecedores e com o planejamento de objetivos mútuos como maneira de assegurar vantagens competitivas. $\mathrm{O}$ termo "troca baseada no relacionamento" (relational-oriented exchanges) é utilizado para denotar relacionamentos duráveis governados primariamente por normas relacionais e princípios éticos com um significado de controle e coordenação. Para adquirir a flexibilidade requerida nas trocas complexas caracterizadas por circunstâncias imprevistas, a troca relacional provê altos níveis de cooperação, planejamento conjunto, e adaptação mútua à necessidade dos parceiros (IZQUIERDO; CILLÁN, 2004).

De acordo com Oliver (1990), as seis principais causas que conduzem ao desenvolvimento de relacionamentos interorganizacionais são:

a) a lei ou regulamento exige empresas para comprar/vender certos produtos e serviços (necessidade);

b) a empresa pode realçar sua reputação (legitimidade);

c) as empresas procuram ligações para exercerem poder e controle sobre outra organização ou seus recursos (assimetria);

d) as empresas querem gerenciar incertezas ambientais (estabilidade);

e) as empresas possuem interesses ou objetivos comuns (reciprocidade);

f) a empresa pode obter baixo custo e alto benefício (eficiência).

Embora estes determinantes possam ser suficientes para a formação de um relacionamento interempresas, eles surgem a partir de múltiplas contingências. Segundo Izquierdo e Cillán (2004), as trocas relacionais são baseadas principalmente na interação das motivações de eficiência, estabilidade e reciprocidade. Com o propósito de aumentar a eficiência e obter estabilidade, uma organização pode decidir por assegurar um relacionamento estável com outra e, além disso, se a organização antecipa uma maior eficiência interna como resultado do relacionamento é desejável perseguir metas mutuamente benéficas, o que possibilita o aumento do interesse mútuo.

Oliver (1990) percebeu que os seis determinantes possuem ligação com condições internas e externas, as quais facilitam ou impedem o estabelecimento do relacionamento. Estas condições estão relacionadas com fatores antecedentes que podem ser classificados em duas categorias:

a) fatores econômicos - referem-se à eficiência e estabilidade, as quais são motivadas pelo desejo de melhorar os resultados econômicos e adaptar a empresa ao ambiente de incertezas. São fatores visíveis quando as empresas dependem uma da outra. A dependência é definida como a necessidade da empresa em manter o relacionamento de maneira a atingir seus objetivos;

b) fatores sociais - referem-se ao objetivo de obter benefícios mútuos pela cooperação, colaboração e coordenação através de ligações afetivas entre as partes. Assim, em acordo com o marketing de relacionamento, a confiança é tomada como variável social principal na explicação da cooperação inter-empresas e dos relacionamentos de longo prazo (MORGAN; HUNT, 1994).

Izquierdo e Cillán (2004) sugerem que as trocas relacionais são conseqüências do grau de interdependência entre as empresas. Dois conceitos são identificados de forma a refletir a variável interdependência: magnitude e assimetria. A magnitude da interdependência é definida como o conjunto total das dependências numa troca, e a assimetria da dependência como o grau comparativo de dependência. Uma alta magnitude de interdependência influencia o estabelecimento de processos e estruturas relacionais porque um relacionamento próximo propicia o uso da força não coercitiva, reduz os conflitos, fomenta a estabilidade e promove a durabilidade, sendo que esta magnitude é moderada pela assimetria. A assimetria produz dois efeitos. Caso haja uma dependência balanceada, ambas as partes terão incentivo para investir no relacionamento, reduzindo o comportamento oportunista. Por outro lado, se os níveis de dependência estão desbalanceados, a parte com menor dependência não terá nenhum incentivo para dividir os seus recursos com a outra em um relacionamento próximo e de longo prazo, pois os benefícios são insignificantes e substituíveis. Mesmo nos casos em que a parte dependente esteja interessada em sustentar um relacionamento orientado ao longo prazo, a parte menos dependente não possuiria interesse em investir em ações conjuntas, e o medo do comportamento oportunista desencorajaria a parte mais dependente a procurar uma posição orientada ao relacionamento próximo e duradouro.

\section{A TERCEIRIZACÃO E O RELACIONAMENTO EMPRESA-TERCEIRIZADO}

O fenômeno da terceirização originou-se nos Estados Unidos, logo após a eclosão da Segunda Guerra Mundial. Hoje, a terceirização configura-se como uma técnica moderna de administração direcionada ao enxugamento das estruturas gerenciais, constituindo-se em uma prática integrante das novas formas de gestão, como a formação de redes organizacionais e de parcerias, que permitem às empresas concentrar-se em suas atividades-fim (ALMEIDA; SILVA, 1999). 
A terceirização é, portanto, uma técnica administrativa que possibilita o estabelecimento de um processo gerenciado de transferência a terceiros, das atividades acessórias e de apoio à sua atividade-fim, permitindo à empresa concentrarse no seu negócio central (QUEIROZ, 1998). A terceirização pode propiciar maior flexibilidade da estrutura de custos da empresa, possibilitando que se mantenham ganhos em curto prazo e investimentos no futuro.

\section{Os fatores relacionais entre empresa-terceirizado}

O terceirizado deve possuir, além de potencial produtivo, o componente relacional. Há diferentes idéias sobre quais fatores são os principais ingredientes do potencial produtivo. Skinner (1978) sugere quatro elementos: preço, qualidade, entrega e flexibilidade, enquanto outros autores adicionam fatores à lista tais como inovação (LEONG et al., 1990), segurança (FERDOWS; DE MEYER, 1990) e serviço (KIM; ARNOLD, 1993). Estes fatores adicionais podem ser considerados como parte dos quatro elementos básicos de Skinner (1978). Por exemplo, serviço como parte da qualidade e entrega, inovação como parte da qualidade e flexibilidade e a segurança como parte da entrega e flexibilidade.

De forma geral, a percepção de quais fatores são os mais importantes varia de acordo com o binômio produto-mercado. Em outras palavras, dependendo do produto ou serviço a ser produzido, com base na respectiva exigência por parte do mercado, diferentes fatores produtivos serão considerados mais importantes.

Os fatores produtivos possuem atributos objetivos, enquanto os fatores relacionais possuem uma característica mais subjetiva, sendo mais complexa a sua determinação.
Os componentes relacionais e suas percepções formam, num contexto inter-organizacional, a base de análise conceitual para a investigação dos relacionamentos interorganizacionais entre empresas contratantes e terceiros.

A definição dos fatores relacionais utilizados como base nesta pesquisa de campo foi feita a partir da identificação, por meio de pesquisa bibliográfica, dos fatores relacionais adequados ao contexto do estudo. Foram selecionados para a investigação das diferenças de percepção entre empresa e terceirizados os fatores comunicação, confiança, cooperação, comprometimento e divisão de riscos/recompensas. $\mathrm{O}$ Quadro 1 contém a informação sobre os as fontes analisadas na determinação dos fatores relacionais.

\section{Comunicação}

Em relacionamentos desenvolvidos, como por exemplo em parcerias, a comunicação e a troca de informações precisam ser claras, francas e abertas em todos os níveis organizacionais através de todas as áreas funcionais. Anderson e Narus (1990, p. 44) definem comunicação como "um compartilhamento formal e informal de informação significativa e oportuna entre as empresas".

O compartilhamento de informações entre os parceiros deve incluir informações técnicas e estratégicas, de maneira a facilitar a tomada de decisão e o planejamento conjunto para encorajar a resolução de problemas conjuntamente (SPEKMAN, 1988) e para reduzir a incerteza, bem como aumentar o controle (MALONI; BENTON, 1997). Uma comunicação aberta também ajuda a construir a confiança mútua e a compreender melhor o negócio do parceiro e sua maneira de trabalho (ELLRAM, 1991). A comunicação

QUADRO 1: As fontes bibliográficas e os fatores do relacionamento.

\begin{tabular}{|c|c|c|}
\hline $\begin{array}{l}\text { FATORES DO } \\
\text { RELACIONAMENTO }\end{array}$ & $\begin{array}{l}\text { COMPONENTE DO } \\
\text { RELACIONAMENTO }\end{array}$ & $\begin{array}{l}\text { ABORDAGEM } \\
\text { TEÓRIGO/EMPÍRIGA }\end{array}$ \\
\hline \multirow{5}{*}{ Fatores Relacionais } & Comunicação & $\begin{array}{l}\text { Anderson e Narus (1990), Metcalf et al. (1992), Morgan e } \\
\text { Hunt (1994), Mohr e Spekman (1994), Lambert et al. (1996), } \\
\text { Abratt e Kelly (2002). }\end{array}$ \\
\hline & Confiança & $\begin{array}{l}\text { Anderson e Narus (1990), Morgan e Hunt (1994), Mohr } \\
\text { e Spekman (1994), Lambert et al. (1996), Abratt e Kelly } \\
\text { (2002). }\end{array}$ \\
\hline & Cooperação & $\begin{array}{l}\text { Anderson e Narus (1990), Metcalf et al. (1992), Morgan e } \\
\text { Hunt (1994), Mohr e Spekman (1994), Lambert et al. (1996); } \\
\text { Sinclair et al. (1996). }\end{array}$ \\
\hline & Comprometimento & $\begin{array}{l}\text { Morgan e Hunt (1994), Mohr e Spekman (1994), Lambert et } \\
\text { al. (1996). }\end{array}$ \\
\hline & $\begin{array}{l}\text { Divisão de Riscos e } \\
\text { Recompensas }\end{array}$ & Ellram (1995), Lambert et al. (1996). \\
\hline
\end{tabular}


entre as partes ajuda o desenvolvimento do relacionamento, cria confiança e fornece informação sobre a necessidade de cada parceiro e conhecimento sobre suas operações, facilitando assim atividades colaborativas e cooperativas (PARVATIYAR; SHETH, 2000).

\section{Confiança}

Anderson e Narus (1990, p. 45) definem a confiança como "a crença da empresa de que outra companhia irá desempenhar ações que resultarão em resultados positivos para ela, bem como não ter ações inesperadas que resultem em resultados negativos".

Segundo Morgan e Hunt (1994), a confiança reduz a probabilidade de término do relacionamento. Confiança e comprometimento fornecem a chave para relacionamentos cooperativos, e de acordo com Ellram e Edis (1996, p. 28), "confiança mútua forma a base para um relacionamento de trabalho forte", ou seja, sem confiança não há um relacionamento verdadeiro de parceria.

\section{m relacionamentos altamente desenvolvidos, os riscos e as recompensas são conjuntamente divididos entre as partes, numa base "ganha-ganha".}

Wiliams apud Nooteboom (1999, p. 56) traça um paralelo entre confiança e cooperação, considerando duas formas principais de ocorrência de cooperação entre duas partes: as formas chamadas de egotista e não-egotista. A primeira baseia-se na coerção ou no medo de sanções de alguma autoridade e pode visar ainda vantagem material ou algum interesse, enquanto a segunda baseia-se em parâmetros éticos como valores, normas de conduta ou ainda laços de amizade, afinidade ou empatia.

Entretanto, Rodrigues (2003) afirma que a confiança baseada em laços de amizade ou em afinidades não é suficiente para embasar a cooperação interfirmas. Da mesma forma, a busca por interesses próprios e a coerção raramente se mostram eficientes para que haja cooperação. Ter confiança é aceitar, ou negligenciar, a probabilidade subjetiva de que o parceiro não utilizará as oportunidades para ter um comportamento oportunista. Assim, a confiança não pode ser imposta, e provavelmente seu desenvolvimento implica em um processo de longo prazo em que os envolvidos desenvolvem, pouco a pouco, relações pessoais e uma reputação para justiça, sendo mais fácil criar confiança da estaca zero do que recuperá-la depois de perdida (RODRIGUES, 2003).

À luz da teoria dos custos de transação, a confiança não é considerada uma componente chave para o desenvolvimento de um relacionamento, sendo considerado o oportunismo como sendo a característica fundamental (WILLIAMSON, 1985). De acordo com a teoria dos custos de transação, contratos escritos e imposições devem ser as práticas preferidas para se lidar com a variável confiança.

Por outro lado, de acordo com a teoria do marketing de relacionamento, confiança e comprometimento são reconhecidos como fatores que afetam claramente o sucesso do relacionamento, sendo determinantes para seu sucesso ou fracasso (MORGAN; HUNT, 1994).

\section{Cooperação}

A cooperação se refere às situações em que as partes trabalham juntas para o atendimento de metas mútuas (ANDERSON; NARUS, 1990). Cooperação e colaboração são dois conceitos que, apesar de algumas pequenas diferenças de interpretação, são tratados pelos autores de maneira relativamente indistinta.

Spekman et al. (1988) afirmam ainda que a cooperação interempresas constrói relações "ganhaganha". Há, porém, distinção entre os termos "cooperação" e "colaboração" e um movimento de amadurecimento natural que vai do primeiro para o segundo. Conforme Mentzer et al. (2001), a cooperação é o "namoro" e a colaboração o "casamento".

De acordo com a teoria dos custos de transação, a cooperação entre os parceiros é focada apenas na transação, isto é, no ato da compra e venda, sendo que o comprador e o vendedor aspiram manter sua independências em todas as áreas de negócio. Já em relação à teoria do marketing de relacionamento, a cooperação é reconhecida como fator importante para o sucesso do relacionamento, sendo refletida na interdependência e interação do relacionamento (PARVATIYAR; SHETH, 2000).

\section{Comprometimento}

De acordo com Dwyer et al. (1987, p.19) "o comprometimento refere-se à promessa implícita ou explícita de continuidade relacional entre os parceiros". Morgan e Hunt (1994) descrevem o comprometimento como o desejo em manter o relacionamento e a confiança em que a outra parte também trabalhará para mantê-la.

Segundo Ellram (1991), uma parceria deve ser construída sobre um forte comprometimento de ambas as partes, e o que é especialmente importante é o comprometimento da alta gerência, além de uma filosofia que encoraje a parceria.

\section{Divisão de Riscos/Recompensas}

Desde que o ambiente de negócios passou a mudar rapidamente, fornecedores bem como compradores sentiram a necessidade de ter um tipo de esquema formal para permitir 
que ambos os parceiros dividam os riscos associados com o ambiente de mudanças. A divisão de riscos, bem como de recompensas, está diretamente ligada aos arranjos cooperativos em que as partes se engajaram. Em relacionamentos desenvolvidos, os riscos e as recompensas são conjuntamente divididos entre as partes, numa base "ganha-ganha" (ANDERSON; NARUS, 1990; SPEKMAN, 1988). Além disso, o desempenho do fornecedor (ou mesmo do relacionamento) é medido de maneira a determinar se os riscos ou recompensas são atribuíveis às falhas ou aos sucessos, respectivamente. Para que o relacionamento caminhe para o longo prazo, deve haver benefícios para ambos os parceiros, e estes benefícios provindos de uma forte colaboração devem naturalmente exceder os benefícios adquiridos se as empresas trabalhassem individualmente. Os benefícios a serem divididos podem vir de diferentes formas, tais como: acesso às novas tecnologias e à informação, acesso a mercados restritos, custos de operação mais baixos tanto para o comprador quanto para o fornecedor, e ainda preços mais baixos (WILSON, 1995).

A teoria dos custos de transação não reconhece a divisão de riscos e recompensas em seus modelos, e a teoria do marketing de relacionamento trata a divisão de riscos e recompensas como importantes para o relacionamento, sendo diretamente relacionada com a satisfação mútua das empresas.

\section{METODOLOGIA DA PESQUISA}

$\mathrm{Na}$ pesquisa de campo aqui apresentada, considera-se o contexto em que uma única empresa contratante terceiriza os serviços de várias empresas terceirizadas concorrentes entre si. Esta deliberação possui um impacto significativo nos resultados da pesquisa, pois o número de compradores de um fornecedor envolvidos na cadeia de suprimento é crucial para a influência na percepção entre os participantes da cadeia. Em uma cadeia de suprimentos que envolve uma empresa contratante e seus terceirizados, os terceirizados estão competindo para melhor servir um comprador monopolístico. Neste ambiente, de acordo com Yan e Gray (1994), o comprador tem maior poder de negociação, já que possui mais alternativas do que os fornecedores.

Nesta pesquisa, optou-se por uma abordagem exploratória com estudo de múltiplos casos numa perspectiva transversal, uma vez que foram observados os fatores para o sucesso do relacionamento da terceirização em um determinado ponto no tempo. O período de estudo considerado é o ano de 2005. O nível de análise é o organizacional, e a unidade de análise, a organização.

A pesquisa foi conduzida em organizações de grande porte com atuação nacional, que possuem atividades ou estão instaladas na cidade de Curitiba/PR ou na sua região metropolitana e que terceirizam total ou parcialmente atividades de serviços por meio de múltiplos terceirizados.

A população da pesquisa constituiu-se de uma empresa contratante do setor industrial metalmecânico, uma do setor financeiro e uma do segmento petroquímico. Para cada uma destas empresas pesquisaram-se cinco empresas terceirizadas, totalizando um conjunto de 18 empresas. Para a seleção dos entrevistados foi adotado um procedimento de caráter intencional por julgamento, já que os membros da amostra foram selecionados para atender a alguns critérios predefinidos, sem a preocupação de aleatoriedade, o que implica, obrigatoriamente, que os resultados encontrados na pesquisa não podem ser generalizados para outras empresas. No entanto, este tipo de pesquisa permite conclusões significativas dada a representatividade das organizações pesquisadas (COOPER; SCHINDLER, 2003).

O Quadro 2 apresenta o número de pessoas entrevistadas nas empresas contratantes $\mathrm{A}, \mathrm{B}$ e $\mathrm{C}$ e nos seus respectivos terceirizados.

Foram entrevistadas pessoalmente 62 pessoas, sendo que 9 pessoas responderam o questionário por correio eletrônico. A coleta dos dados primários ocorreu através das informações obtidas com funcionários das empresas contratantes e terceirizadas que mantêm um inter-relacionamento direto através de questionários com perguntas fechadas.

Com a intenção de não provocar distorções na pesquisa, foram dados aos entrevistados esclarecimentos acerca do significado de cada fator de relacionamento. Quando a in-

Quadro 2: Número de entrevistados nas empresas e em seus terceirizados.

\begin{tabular}{|c|c|c|c|c|c|c|c|}
\hline \multirow{2}{*}{ EMPRESA } & \multirow{2}{*}{ SETOR } & NÚMERO DE ENTREVISTADOS & \multicolumn{4}{|c|}{ NÚMERO DE ENTREVISTADOS } \\
\cline { 4 - 8 } & & EMPRESA CONTRATANTE & T1 & T2 & T3 & T4 & T5 \\
\hline A & Metalmecânico & 10 & 3 & 3 & 3 & 3 & 3 \\
\hline B & Financeiro & 7 & 4 & 4 & 4 & 4 & 4 \\
\hline C & Petroquímico & 4 & 3 & 3 & 3 & 3 & 3 \\
\hline
\end{tabular}


formação foi obtida por meio eletrônico, os esclarecimentos foram feitos via contato telefônico.

No entanto, é importante salientar que por mais cuidado que se tenha tido na exposição para os entrevistados do significado dos fatores avaliados, não é possível descartar a possibilidade de discrepância no entendimento de um mesmo termo entre o pesquisador e o entrevistado. O fato de se analisar as respostas de mais de um respondente por empresa tenta minimizar estatisticamente esta questão. empresas contratantes e terceirizadas, respectivamente.

\section{RESULTADOS}

Foram coletados os dados utilizando-se formulários contendo as questões apresentadas no Quadro 3. As respostas obtidas em cada empresa foram tabuladas, e os resultados da tabulação são resumidos na Tabela 1 .

Os valores apresentados na tabela são referentes à média das respostas obtidas nos questionários, representando um valor médio dos fatores chave do relacionamento percebido tanto pela empresa contratante, quanto pela empresa terceirizada.

\section{evolução, cuja principal característica é a mudança da lógica do produto para a lógica da integração/cooperação.}

Os dados da empresa A (setor metalmecânico) foram obtidos através de entrevistas realizadas na empresa com os responsáveis pela contratação de serviços técnicos (duas pessoas), com os supervisores diretos dos terceirizados (seis pessoas), e com os supervisores diretos (duas pessoas). Com relação aos terceirizados da empresa $\mathrm{A}$, foram realizadas entrevistas com o sócio ou supervisor de cada empresa terceirizada (uma pessoa), e com os responsáveis diretos pela execução dos serviços técnicos (duas pessoas por empresa).

Os dados da empresa B (setor financeiro) foram obtidos através de informações solicitadas por correio eletrônico à área responsável pela contratação de terceirizados (três pessoas), informações solicitadas por correio eletrônico aos supervisores responsáveis pelas empresas terceirizadas (três pessoas), e entrevista realizada na empresa com o gerente do setor (uma pessoa). Com relação aos terceirizados da empresa $\mathrm{B}$, foram realizadas entrevistas com o sócio ou supervisor de cada empresa terceirizada (uma pessoa), e com os responsáveis diretos pela execução dos serviços (três pessoas por empresa).

Os dados da empresa $\mathrm{C}$ (setor petroquímico) foram obtidos através de entrevista realizada na empresa com um dos supervisores diretos dos terceirizados (uma pessoa), e informações solicitadas por correio eletrônico a supervisores diretos (três pessoas). Com relação aos terceirizados da empresa $\mathrm{C}$, foram realizadas entrevistas com o sócio ou supervisor de cada empresa terceirizada (uma pessoa), e com os responsáveis diretos pela execução dos serviços técnicos (duas pessoas por empresa).

No Quadro 3, são apresentadas as questões avaliadas em uma escala de um a sete pontos para as respostas e nos apêndices $\mathrm{A}$ e $\mathrm{B}$ são apresentados os questionários aplicados às

\section{Análise estatística utilizada}

Para comparar os resultados dos três conjuntos de empresas pesquisadas foi empregado o método da Análise de Variância - ANOVA (ARA et al., 2003). Utilizou-se a chamada análise da variância de um critério, uma vez que os grupos foram categorizados em função de uma única característica, ou critério, que neste estudo corresponde a cada um dos fatores de relacionamento.

Ao comparar as médias dos resultados obtidos em cada uma das empresas e seus respectivos terceirizados, são testadas duas hipóteses:

H0: não existe diferença entre as médias (hipótese nula);

H1: pelo menos uma das médias é diferente das demais (hipótese alternativa).

O teste F (WINTER, 2002) é utilizado para verificar se a variabilidade dos dados é decorrente do fato de que os mesmos pertencem a diferentes contextos de avaliação ou se a variabilidade é devida ao acaso (variabilidade residual).

A partir dessa análise é possível determinar se a hipótese nula deve ou não ser rejeitada. Se a hipótese nula for rejeitada, isto indica que as médias não são iguais, entretanto não é possível determinar quais são as médias estatisticamente diferentes das demais.

Nestes casos, além da análise de variância, foi aplicado o teste $\mathrm{Z}$ bilateral (WINTER, 2002) para determinar a diferença entre duas médias com desvios padrões conhecidos (média da respostas da empresa e média das respostas dos terceirizados). O nível de significância considerado, $\alpha$, foi de $5 \%$.

\section{Análise dos dados}

$\mathrm{Na}$ Tabela 2, são apresentados os dados coletados da Empresa A e seus terceirizados, além da análise estatística realizada. 
Das seis empresas (Empresa A + cinco empresas terceirizadas), foram pesquisadas 25 pessoas (10 da empresa $\mathrm{A} \mathrm{e}$ 15 das empresas terceirizadas).

Objetivando analisar se há diferenças entre as médias, foi realizado o teste $\mathrm{F}$ nas amostras. Pela análise dos dados apresentados na Tabela 2, nota-se que os fatores de relacionamento comunicação, confiança e divisão de risco/ oportunidade, apresentaram diferenças significativas de percepção entre as empresas $(\mathrm{F}(\mathrm{x})>\mathrm{F}(\alpha))$.

Através da análise dos resultados do teste $\mathrm{Z}$ bilateral evidenciam-se as diferenças entre a média das respostas da empresa A e dos seus terceirizados, indicando as diferenças (lacunas ou gaps) de percepção entre essas partes $(\mathrm{Z}(\mathrm{x})>\mathrm{Z}(\alpha))$.

A última coluna da Tabela 2 apresenta, em valores numéricos, o gap de percepção entre a empresa e seus terceirizados, para cada um dos fatores de relacionamento analisados (diferença entre a média da empresa A e a média das respostas de seus terceirizados).

Pode ser observado que para a empresa A, a média das respostas dos funcionários entrevistados (10 pessoas) em relação à percepção quanto ao grau de importância do fator comunicação foi de 3,30 , enquanto que a média das respostas dos funcionários das empresas terceirizadas (15 pessoas) foi de 5,8. Assim, a lacuna (gap) de percepção encontrada em relação a este fator foi de $2,50(5,8-3,3)$. $\mathrm{O}$ fator comunicação possui uma atribuição de importância maior do ponto de vista dos terceirizados do que do ponto de vista da empresa contratante.

Analogamente, observa-se com relação ao fator confiança um gap de 1,53. Neste caso, este fator possui uma atribuição de importância maior do ponto de vista da empresa

Quadro 3: Fatores do relacionamento e as questões avaliadas.

\begin{tabular}{|c|c|c|}
\hline $\begin{array}{l}\text { FATORES DO } \\
\text { RELACIONAMENTO }\end{array}$ & $\begin{array}{l}\text { COMPONENTE DO } \\
\text { RELAGIONAMENTO }\end{array}$ & $\begin{array}{l}\text { QUESTÕES (AVALIAÇÃO EM UMA ESCALA DE SETE } \\
\text { PONTOS) }\end{array}$ \\
\hline \multirow{10}{*}{$\begin{array}{l}\text { FATORES } \\
\text { RELACIONAIS } \\
\text { (Questões fechadas) }\end{array}$} & \multirow{2}{*}{$\begin{array}{l}\text { COMUNICAÇÃO } \\
\text { Escala: } \\
1 \text { = pouco importante } \\
7 \text { = muito importante }\end{array}$} & $\begin{array}{l}\text { Baseado em suas experiências passadas e presentes, qual } \\
\text { a importância da comunicação para o relacionamento de } \\
\text { trabalho com as empresas terceirizadas? }\end{array}$ \\
\hline & & $\begin{array}{l}\text { Baseado em suas experiências passadas e presentes, qual } \\
\text { a importância da comunicação para o relacionamento de } \\
\text { trabalho com a empresa contratante? }\end{array}$ \\
\hline & \multirow{2}{*}{$\begin{array}{l}\text { CONFIANÇA } \\
\text { Escala: } \\
1 \text { = é pouco importante } \\
7 \text { = é muito importante }\end{array}$} & $\begin{array}{l}\text { Baseado em suas experiências passadas e presentes, como } \\
\text { você caracteriza o nível de confiança que sua empresa } \\
\text { possui no relacionamento de trabalho com o terceirizado? }\end{array}$ \\
\hline & & $\begin{array}{l}\text { Baseado em suas experiências passadas e presentes, como } \\
\text { você caracteriza o nível de confiança que sua empresa } \\
\text { possui no relacionamento de trabalho com a empresa } \\
\text { contratante? }\end{array}$ \\
\hline & \multirow{2}{*}{$\begin{array}{l}\text { COOPERAÇÃO } \\
\text { Escala: } \\
1 \text { = concorda fortemente } \\
7 \text { = discorda fortemente }\end{array}$} & $\begin{array}{l}\text { Minha empresa acredita que a cooperação é fundamental } \\
\text { para o relacionamento com o terceirizado. }\end{array}$ \\
\hline & & $\begin{array}{l}\text { Minha empresa acredita que a cooperação é fundamental } \\
\text { para o relacionamento com a empresa contratante. }\end{array}$ \\
\hline & \multirow{2}{*}{$\begin{array}{l}\text { COMPROMETIMENTO } \\
\text { Escala: } \\
1 \text { = concorda fortemente } \\
7 \text { = discorda fortemente }\end{array}$} & $\begin{array}{l}\text { Minha empresa acredita que estar comprometido com o } \\
\text { terceirizado é fundamental para o relacionamento. }\end{array}$ \\
\hline & & $\begin{array}{l}\text { Minha empresa acredita que estar comprometido com a } \\
\text { empresa contratante é fundamental para o relacionamento. }\end{array}$ \\
\hline & \multirow{2}{*}{$\begin{array}{l}\text { DIVISÃO DE RISCOS E } \\
\text { RECOMPENSAS } \\
\text { Escala: } \\
1 \text { = discorda fortemente } \\
7 \text { = concorda fortemente }\end{array}$} & $\begin{array}{l}\text { Os riscos e as recompensas devem ser igualmente divididos } \\
\text { entre nossa empresa e o terceirizado. }\end{array}$ \\
\hline & & $\begin{array}{l}\text { Os riscos e as recompensas devem ser igualmente divididos } \\
\text { entre nossa empresa e a empresa contratante. }\end{array}$ \\
\hline
\end{tabular}


contratante, ou seja, a empresa que contrata percebe uma importância maior neste fator do que aquela percebida pelos terceirizados.

O fator de relacionamento divisão de riscos/recompensas obteve uma lacuna (gap) de percepção com um valor de 2,13 . Pode-se observar que do ponto de vista dos terceirizados há uma maior concordância com a divisão dos riscos/recompensas do que do ponto de vista da empresa contratante.

De maneira análoga, esta análise foi realizada para as empresas B e C. As Tabelas 3 e 4 resumem os resultados para estas empresas.

Para a empresa B e seus terceirizados, os gaps de percepção encontram-se nos fatores comunicação, confiança e divisão de riscos/recompensas.

O fator comunicação apresentou uma lacuna de percepção de 1,70. Tal qual no caso da empresa A, este fator possui uma importância maior na percepção dos terceirizados.

A lacuna (gap) de percepção encontrada em relação ao fator confiança foi de 1,40. Da mesma forma que para a empresa A, este fator possui uma atribuição de importância maior do ponto de vista da empresa contratante do que do ponto de vista dos terceirizados.
O fator de relacionamento divisão de riscos/recompensas obteve uma lacuna ( $g a p$ ) de percepção com um valor de 1,52. Aqui este fator possui uma atribuição de concordância maior do ponto de vista dos terceirizados, da mesma forma que o observado para a empresa A.

Da mesma forma que para a empresa A não há diferença significativa, ao nível de significância de 5\%, entre as percepções da empresa B e seus terceirizados com relação aos fatores cooperação e comprometimento.

Os resultados da análise realizada nas respostas da empresa contratante $\mathrm{C}$ e seus terceirizados apontaram gaps de percepção nos fatores: comunicação, cooperação e divisão dos riscos/recompensas.

O fator comunicação obteve um gap de percepção de 1,50. Aqui também este fator possui uma importância maior na percepção dos terceirizados, tal qual o ocorrido com as empresas A e B.

Com relação ao fator cooperação, o gap de percepção foi de 2,00 pontos, sendo maior a importância do ponto de vista da empresa $\mathrm{C}$ do que do ponto de vista de seus terceirizados.

Também para a empresa $\mathrm{C}$ há um gap de percepção em relação ao fator divisão de riscos/ recompensas entre a Empresa $\mathrm{C}$ e seus terceirizados de 1,57 ponto, tendo este

Tabela 1: Respostas das empresas contratantes e terceirizados.

\begin{tabular}{|c|c|c|c|c|}
\hline $\begin{array}{c}\text { EMPRESA A } \\
\text { (SETOR METALMECÂNICO) }\end{array}$ & $\begin{array}{c}\text { MÉDIA EMPRESA } \\
\text { A }\end{array}$ & $\begin{array}{l}\text { DESVIO } \\
\text { PADRÃOO }\end{array}$ & $\begin{array}{c}\text { MÉDIA DOS } \\
\text { TERCEIRIZADOS A }\end{array}$ & $\begin{array}{l}\text { DESVIO } \\
\text { PADRÃOO }\end{array}$ \\
\hline Comunicação & 3,30 & 1,49 & 5,80 & 1,61 \\
\hline Confiança & 4,80 & 1,32 & 3,27 & 1,03 \\
\hline Cooperação & 4,40 & 1,58 & 4,27 & 1,62 \\
\hline Comprometimento & 3,90 & 2,13 & 4,73 & 2,02 \\
\hline Divisão de riscos/Recomp. & 3,80 & 1,81 & 5,93 & 0,88 \\
\hline $\begin{array}{c}\text { EMPRESA B } \\
\text { (SETOR FINANCEIRO) }\end{array}$ & $\begin{array}{c}\text { MÉDIA EMPRESA } \\
\text { B }\end{array}$ & $\begin{array}{l}\text { DESVIO } \\
\text { PADRÃOO }\end{array}$ & $\begin{array}{c}\text { MÉDIA DOS } \\
\text { TERGEIRIZADOS B }\end{array}$ & $\begin{array}{l}\text { DESVIO } \\
\text { PADRÃOO }\end{array}$ \\
\hline Comunicação & 4,00 & 1,73 & 5,70 & 1,03 \\
\hline Confiança & 6,00 & 1,15 & 4,60 & 1,88 \\
\hline Cooperação & 4,86 & 1,68 & 4,50 & 1,91 \\
\hline Comprometimento & 4,71 & 1,50 & 5,80 & 0,95 \\
\hline Divisão de riscos/Recomp. & 3,43 & 1,51 & 4,95 & 1,73 \\
\hline $\begin{array}{c}\text { EMPRESA C } \\
\text { (SETOR PETROQUÍMICO) }\end{array}$ & $\begin{array}{c}\text { MÉDIA EMPRESA } \\
\text { C }\end{array}$ & $\begin{array}{l}\text { DESVIO } \\
\text { PADRÃOO }\end{array}$ & $\begin{array}{c}\text { MÉDIA DOS } \\
\text { TERCEIRIZADOS C }\end{array}$ & $\begin{array}{l}\text { DESVIO } \\
\text { PADRÃOO }\end{array}$ \\
\hline Comunicação & 3,50 & 0,58 & 5,00 & 1,31 \\
\hline Confiança & 4,50 & 1,73 & 4,53 & 1,73 \\
\hline Cooperação & 6,00 & 0,82 & 4,00 & 1,00 \\
\hline Comprometimento & 5,25 & 2,36 & 5,00 & 1,77 \\
\hline Divisão de riscos/Recomp. & 3,50 & 1,29 & 5,07 & 1,53 \\
\hline
\end{tabular}


fator, da mesma forma que para as outras empresas, maior importância para os terceirizados.

Dessa maneira, as diferenças de percepção nestes três casos são apresentadas resumidamente na Tabela 5.

Nos três casos estudados, os fatores de relacionamento comunicação e divisão de riscos/recompensas apresentaram lacunas (gaps) de percepção entre as empresas contratantes e seus terceirizados, demonstrando maior concordância do ponto de vista dos terceirizados do que do ponto de vista das empresas contratantes.

Para as empresas A e B o fator confiança, que apresentou um gap de 2,93 pontos, apresentando uma atribuição de importância maior do ponto de vista das empresas A e B do que do ponto de vista de seus terceirizados.

É coincidente nos três casos, que o fator de relacionamento comprometimento não apresentou diferença significativa, ao nível de significância de $5 \%$, entre as percepções das empresas e seus terceirizados. Ambos, empresas e terceirizados, percebem e atribuem um grau de importância muito próximo, sendo que o gap de percepção neste caso pode ser considerado nulo. Também o fator cooperação não apresentou diferença significativa, ao nível de significância de 5\%,

Tabela 2: Análise Empresa A e seus Terceirizados.

\begin{tabular}{|c|c|c|c|c|c|c|c|c|c|}
\hline $\begin{array}{l}\text { EMPRESA A } \\
\text { (SETOR } \\
\text { METALMECÂNICO) }\end{array}$ & $\begin{array}{l}\text { MÉDIA } \\
\text { EMPRESA } \\
\text { A }\end{array}$ & $\begin{array}{l}\text { DESVIO } \\
\text { PADRÃOO }\end{array}$ & $\begin{array}{l}\text { MÉDIA DOS } \\
\text { TERGEIRIZADOS } \\
\text { A }\end{array}$ & $\begin{array}{l}\text { DESVIOO } \\
\text { PADRÃOO }\end{array}$ & $\begin{array}{l}\text { TESTE F } \\
\text { F(x) }\end{array}$ & $F(\alpha)$ & $\begin{array}{l}\text { TESTE } \mathbf{Z} \\
\mathbf{Z}(x)=0\end{array}$ & $\mathbf{Z}(\alpha)$ & $\begin{array}{l}\text { GAP } \\
\text { EMPRESA } \\
\text { TERC. }\end{array}$ \\
\hline Comunicação & 3,30 & 1,49 & 5,80 & 1,61 & 2,88 & 2,74 & $-3,97$ & 1,96 & 2,50 \\
\hline Confiança & 4,80 & 1,32 & 3,27 & 1,03 & 2,83 & 2,74 & 3,10 & 1,96 & 1,53 \\
\hline Cooperação & 4,40 & 1,58 & 4,27 & 1,62 & 0,80 & 2,74 & 0,20 & 1,96 & 0,13 \\
\hline Comprometimento & 3,90 & 2,13 & 4,73 & 2,02 & 2,71 & 2,74 & $-0,98$ & 1,96 & 0,83 \\
\hline $\begin{array}{l}\text { Divisão de riscos/ } \\
\text { Recomp. }\end{array}$ & 3,80 & 1,81 & 5,93 & 0,88 & 2,82 & 2,74 & $-3,46$ & 1,96 & 2,13 \\
\hline
\end{tabular}

Tabela 3: Análise empresa B e seus terceirizados.

\begin{tabular}{|c|c|c|c|c|c|c|c|c|c|}
\hline $\begin{array}{c}\text { EMPRESA B } \\
\text { (SETOR FINANCEIRO) }\end{array}$ & $\begin{array}{c}\text { MÉDIA } \\
\text { EMPRESA } \\
\text { B }\end{array}$ & $\begin{array}{l}\text { DESVIO } \\
\text { PADRÃ̃O }\end{array}$ & $\begin{array}{c}\text { MÉDIA DOS } \\
\text { TERCEIRIZADOS } \\
\text { B }\end{array}$ & $\begin{array}{l}\text { DESVIO } \\
\text { PADRÃOO }\end{array}$ & $\begin{array}{l}\text { TESTE F } \\
F(x)\end{array}$ & $F(\alpha)$ & $\begin{array}{l}\text { TESTE } \mathbf{Z} \\
\mathbf{Z}(\mathbf{x})=\mathbf{0}\end{array}$ & $\mathbf{z}(\alpha)$ & $\begin{array}{l}\text { GAP } \\
\text { EMPRESA/ } \\
\text { TERC. }\end{array}$ \\
\hline Comunicação & 4,00 & 1,73 & 5,70 & 1,03 & 2,89 & 2,68 & $-2,45$ & 1,96 & 1,70 \\
\hline Confiança & 6,00 & 1,15 & 4,60 & 1,88 & 4,95 & 2,68 & 2,31 & 1,96 & 1,40 \\
\hline Cooperação & 4,86 & 1,68 & 4,50 & 1,91 & 0,27 & 2,68 & 0,47 & 1,96 & 0,36 \\
\hline Comprometimento & 4,71 & 1,50 & 5,80 & 0,95 & 2,25 & 2,68 & $-1,80$ & 1,96 & 1,09 \\
\hline $\begin{array}{l}\text { Divisão de riscos/ } \\
\text { Recomp. }\end{array}$ & 3,43 & 1,51 & 4,95 & 1,73 & 2,82 & 2,68 & $-2,20$ & 1,96 & 1,52 \\
\hline
\end{tabular}

Tabela 4: Análise empresa $C$ e seus terceirizados.

\begin{tabular}{|c|c|c|c|c|c|c|c|c|c|}
\hline $\begin{array}{c}\text { EMPRESA C } \\
\text { [SETOR } \\
\text { PETROQuímico) }\end{array}$ & $\begin{array}{c}\text { MÉDIA } \\
\text { EMPRESA } \\
\text { C }\end{array}$ & $\begin{array}{l}\text { DESVIO } \\
\text { PADRÃOO }\end{array}$ & $\begin{array}{l}\text { MÉDIA DOS } \\
\text { TERCEIRIZADOS } \\
\text { C }\end{array}$ & $\begin{array}{l}\text { DESVIO } \\
\text { PADRÃOO }\end{array}$ & $\begin{array}{c}\text { TESTE F } \\
F(x)\end{array}$ & $F(\alpha)$ & $\begin{array}{l}\text { TESTE Z } \\
\mathbf{Z}(\mathrm{x})=0\end{array}$ & $\mathbf{z}(\alpha)$ & $\begin{array}{c}\text { GAP } \\
\text { EMPRESA/ } \\
\text { TERC. }\end{array}$ \\
\hline Comunicação & 3,50 & 0,58 & 5,00 & 1,31 & 3,22 & 3,03 & $-3,37$ & 1,96 & 1,50 \\
\hline Confiança & 4,50 & 1,73 & 4,53 & 1,73 & 1,66 & 3,03 & $-0,03$ & 1,96 & 0,03 \\
\hline Cooperação & 6,00 & 0,82 & 4,00 & 1,00 & 3,60 & 3,03 & 4,14 & 1,96 & 2,00 \\
\hline Comprometimento & 5,25 & 2,36 & 5,00 & 1,77 & 0,37 & 3,03 & 0,20 & 1,96 & 0,25 \\
\hline $\begin{array}{l}\text { Divisão de riscos/ } \\
\text { Recomp. }\end{array}$ & 3,50 & 1,29 & 5,07 & 1,53 & 3,06 & 3,03 & $-2,07$ & 1,96 & 1,57 \\
\hline
\end{tabular}


entre as percepções das empresas A e B e seus terceirizados. Por sua vez, o fator confiança não apresentou diferença significativa, ao nível de significância de 5\%, entre as percepções da empresa $\mathrm{C}$ e seus terceirizados.

Assim, através da identificação e análise das lacunas de percepção entre empresas e terceirizados, é possível indicar oportunidades de melhoria no sistema de gestão do relacionamento interempresas. Ações visando melhorar a percepção, principalmente em relação aos fatores apresentados no quadro abaixo, podem contribuir para um relacionamento mais próximo e produtivo baseado na compreensão mútua entre as respectivas empresas e seus terceirizados.

A Tabela 6 apresenta em ordem de importância os fatores com maiores lacunas (gaps) percebidas entre empresas e terceirizados. Para as empresas A e B, a primeira oportunidade de melhoria é em relação ao fator comunicação, e para a empresa $\mathrm{C}$ o fator cooperação surge como primeira oportunidade de melhoria.

\section{CONCLUSÕES}

Diante dos resultados obtidos nesta pesquisa, é possível chegar a algumas conclusões, e a partir delas efetuar algumas recomendações indicando oportunidades de melhoria na gestão do inter-relacionamento entre empresas e terceirizados.

Considera-se que a metodologia adotada nesta pesquisa permitiu analisar os dois lados das percepções sobre relacionamento (contratante e terceirizado), além de envolver mais de um informante por empresa, o que possibilitou produzir um quadro mais compreensivel e significativo dos relacionamentos estudados.

Foram identificadas lacunas significativas nos fatores de relacionamento comunicação (empresas A, B e C), divisão de riscos/recompensas (empresas A, B e C), confiança (empresas A e B) e cooperação (empresa $\mathrm{C}$ ).

A identificação e análise das lacunas de percepção encontradas nos três estudos de caso permitem identificar oportunidades de melhoria da gestão do relacionamento entre empresas contratantes e terceirizados. Para as empresas A e B, a primeira oportunidade de melhoria identificada foi em relação ao fator comunicação. Para a empresa $\mathrm{C}$, o fator cooperação foi identificado como primeira oportunidade de melhoria.

A sinalização para que as empresas contratantes reforcem seus canais de comunicação e revejam como estão se dando com seus terceirizados alinha-se com o proposto por Parvatiyar e Shieth (2000) que pregam que no caso das parcerias, a

Tabela 5: Lacunas de percepção.

\begin{tabular}{|l|c|c|c|}
\hline \multicolumn{1}{|c|}{} & $\begin{array}{c}\text { EMPRESA } \\
\text { A }\end{array}$ & $\begin{array}{c}\text { EMPRESA } \\
\text { B }\end{array}$ & $\begin{array}{c}\text { EMPRESA } \\
\text { C }\end{array}$ \\
\hline Comunicação & Gap & Gap & Gap \\
\hline Confiança & Gap & Gap & Gap \\
\hline Cooperação & & & \\
\hline Comprometimento & & & Gap \\
\hline Divisão de riscos/Recomp. & Gap & Gap & \\
\hline
\end{tabular}

Tabela 6: Principais oportunidades de melhoria para as empresas A, B e C.

\begin{tabular}{|c|l|r|}
\hline EMPRESA & \multicolumn{1}{|c|}{ PRINCIPAIS OPORTUNIDADES DE MELHORIA } & GAP \\
\hline \multirow{2}{*}{ A } & 1- Comunicação & 2,50 \\
& 2- Divisão de riscos / recompensas & 2,13 \\
& 3- Confiança & 1,53 \\
\hline \multirow{2}{*}{ B } & 1- Comunicação & 1,70 \\
& 2- Divisão de riscos / recompensas & 1,52 \\
\hline C & 3- Confiança & 1,40 \\
\hline & 1- Cooperação & 2,00 \\
\hline
\end{tabular}


comunicação deve abranger não somente aspectos técnicos, mas também visar o desenvolvimento do relacionamento, criando a confiança que fomenta atividades colaborativas e cooperativas. Assim, no relacionamento entre as empresas A e B e seus terceirizados, a comunicação e a troca de informações podem ser melhoradas de maneira a se tornarem claras, francas e abertas através de todas as áreas funcionais. A comunicação pode incluir informações técnicas e estratégicas, facilitando a tomada de decisão e o planejamento conjunto, além da redução da incerteza e do aumento do controle (MALONI; BENTON, 1997). Uma comunicação aberta também ajudará a construir a confiança mútua e a compreender melhor o negócio do parceiro e sua maneira de trabalho (ELLRAM, 1991).

Quanto ao relacionamento entre a empresa $\mathrm{C}$ e seus terceirizados, a cooperação pode estar focada apenas na transação, isto é, no ato da compra e venda. Isto pode estar causando o gap de percepção em relação a este fator. Um aumento na interdependência e na interação do relacionamento pode melhorar as situações em que as partes trabalham juntas para o atendimento de metas mútuas construindo um relacionamento cooperativo.

Para as três empresas, a segunda oportunidade para melhoria é em relação ao fator de relacionamento divisão de riscos/recompensas. Este fator recebeu, nos três estudos de caso, um grau de concordância maior do ponto de vista dos terceirizados, ou seja, os terceirizados perceberam este fator como crítico para um bom relacionamento e concordam mais fortemente com a divisão igualitária dos riscos e recompensas entre terceirizados e empresas.

Isto dá indícios de que os terceirizados se reconhecem como a parte frágil da relação, que nestes casos é evidente. A assimetria de poder normalmente é verificada nas terceirizações e possivelmente também nos casos estudados nesta pesquisa (as empresas contratantes estudadas são de grande porte e líderes de seus setores). Esta assimetria de poder acaba por prejudicar o relacionamento, uma vez que gera insegurança na parte mais fraca e dependente.

Assim o fator divisão de riscos/recompensas surge como oportunidade para melhoria em todas as empresas pesquisadas. Os terceirizados podem estar percebendo uma divisão não uniforme, com uma absorção maior de sua parte dos riscos envolvidos e menor das recompensas. Este gap de percepção pode ser reduzido através de um esquema formal para permitir que ambos os parceiros dividam os riscos, bem como as recompensas, associadas com o ambiente de mudanças. O desempenho dos terceirizados, ou mesmo do relacionamento entre contratantes e terceirizados, pode ser medido de maneira a determinar se os riscos ou recompen- sas são atribuíveis às falhas ou sucessos respectivamente, de maneira que ocorra a divisão das recompensas ou a absorção dos riscos. Visando um relacionamento de parceria, que caminhe para o longo prazo, deve haver benefícios para ambos os parceiros, e estes benefícios devem naturalmente exceder os benefícios que seriam adquiridos se as empresas trabalhassem individualmente.

\section{os três casos estudados, os fatores de cionamento comunicação e divisão de riscos/recompensas apresentaram lacunas (gaps) de percepção entre as empresas contratantes e seus terceirizados.}

É possível concluir que os resultados da pesquisa demonstram claramente que, à luz deste estudo, as empresas nem sempre "falam a mesma língua" quando mantêm um relacionamento com seus terceirizados. A investigação das lacunas (gaps) de percepção no relacionamento entre contratante e fornecedor, através da participação de ambas as empresas com múltiplos informantes, identificou diferenças e semelhanças entre os casos escolhidos. Isto evidencia que os fatores críticos de sucesso mais importantes, considerados fatores-chave para o sucesso do relacionamento, percebidos pelos terceirizados, nem sempre são os mesmos que os percebidos pela empresa contratante. Estas diferenças de percepção entre o ponto de vista da empresa e do terceirizado provocam um desalinhamento das expectativas das empresas envolvidas, o que pode prejudicar a cooperação, e as parcerias interorganizacionais.

Finalmente, observou-se que a identificação e estudo das lacunas de percepção permitem um esclarecimento de quais são os fatores considerados críticos para um relacionamento de terceirização com sucesso, indicando para as partes envolvidas que aspectos devem ser desenvolvidos ou podem ser melhorados.

A situação que se apresenta nesta pesquisa é de interação dinâmica e, muitas vezes contraditória entre empresas contratantes e contratadas, que podem chegar a bom termo, com a consciência, aceitação e entendimento dos interesses individuais a serem conciliados, tendo-se em vista o objetivo comum de melhor condição competitiva e o compartilhamento justo de riscos, custos e recompensas. Neste sentido, a metodologia de avaliação de lacunas de relacionamento utilizada neste trabalho pode servir como uma ferramenta viável para avaliação de outros relacionamentos interempresariais.

Deixa-se como sugestão para a complementação deste trabalho, ou de outro do gênero, a inclusão de um viés 
qualitativo na pesquisa, através de entrevistas estruturadas, porém com questões abertas, de maneira a capturar aspectos subjetivos do relacionamento entre contratantes e terceirizados.

Por fim, como recomendação para pesquisas futuras fica a sugestão à busca de uma resposta para a pergunta: Se a realidade da gestão dos relacionamentos entre empresas e terceirizados não corresponde ipsis litteris virgulisque àquilo que as teorias acadêmicas de relacionamento dizem que deveria ser, o que então as pessoas que tomam decisões nas empresas estão fazendo de fato, e por que o fazem assim?

\section{Artigo recebido em 20/03/2006 \\ Aprovado para publicação em 11/04/2007}

\section{- Referências}

ABRATT, R.; KELLY, P. M. Customer-supplier partnerships - Perceptions of a successful key account management program. Industrial Marketing Management, $\mathrm{v}$. 31 , n. 5, p. 467-476, 2002

ALMEIDA, M. C.; SILVA, R. A. R. Estratégias de Terceirização: um estudo em grandes empresas mineiras. In: XXIII Encontro Nacional da Associação Nacional dos Programas de Pós-Graduação em Administração, 1999, Foz do Iguaçu. Anais... Rio de Janeiro: Anpad, 1999. 1 CD-ROM.

ANDERSON, J.; NARUS, A. A model for distribution firm and manufacturer firm working partnerships. Journal of Marketing, v. 54, n.1, p. 42-58, jan. 1990.

ARA, A. B.; MUSETTI, A. V.; SCHNEIDERMAN, B. Introdução à Estatística. São Paulo: Instituto Mauá de Tecnologia, Edgard Blucher Ltda, 2003.

BRASIL, H. G. A Empresa e a Estratégia da Terceirização. Revista de Administração de Empresas, São Paulo, v. 33, n. 2, p. 6-11, mar./abr. 1993.

COOPER, D. R. e SCHINDLER, P. S. Métodos de Pesquisa em Administração. Trad. Luciana de Oliveira da Rocha. Porto Alegre: Bookman, 2003.

DONEY, P. M.; CANNON, J. P. An Examination of the Nature of Trust in Buyer-Seller Relationships. Journal of Marketing, v. 61-62, p. 35-51, 1997.

DWYER, F. R.; SCHURR, P. H.; OH, S. Developing Buyer-Seller Relationships. Journal of Marketing, v. 51, n. 1, p. 11-27, abr. 1987.
ELLRAM, L. M. Key Success Factors and Barriers in International Purchasing Partnerships. Management Decision, v. 29 p. $38-44,1991$

A managerial guideline for the development and implementation of purchasing partnerships. tation of purchasing partnerships. Materials Management, v. 31, n. 2, p. 10-16, 1995.

ELLRAM, L. M.; EDIS, O. R. V. A Case Study of Successfull Partnering Implementation. International Journal of Purchasing and Materials Management, v. 32 , p. 20-28, 1996.

FARIA, A. Terceirização: um desafio para o movimento sindical. In: MARTINS, Heloísa de Souza; RAMALHO, J. R. (Orgs.). Terceirização: diversidade e negociação no mundo do trabalho. São Paulo: Hucitec/Cedi-Nets, p. 41-61, 1994.

FERDOWS, K.; DE MEYER, A. Lasting improvements in manufacturing performance: in search of a new theory. Journal of Operations Management, v. 9, n. 2, p. 168-185, 1990.

GRÖNROOS, C. Marketing: gerenciamento de serviços. A competição por serviços na hora da verdade. 14. ed. Tradução por Cristina Bazán. Rio de Janeiro: Campus, 1993

IZQUIERDO, C.; CILLÁN, J. The interaction of dependence and trust in long-term industrial relationships. European Journal of Marketing, v. 38, n. 8 p. 974-994, 2004.

KIM, J. S.; ARNOLD, P., Manufacturing competence and business performance: a framework and empirical analysis. International Journal of Operations $\&$ Production Management, v. 13, n. 10 1993.
LAMBERT, D. M.; EMMELHANIZ, M. A.; GARDNER, J. T. Developing and implementing Supply Chain Partnerships. The International Journal of Logistics Management. U. S. A., v. 7, n. 2, p. 117, 1996.

LEITE, J. C. Terceirização em informática. São Paulo: Makron Books, 1994.

LEONG, G. K. SNYDER, D. L. WARD, P. T. Research in the process and content of manufacturing strategy. Omega, v. 18, n. 2, p. 109-122, 1990

MALONI, M. J.; BENTON, W. C. Supply Chain Partnerships: Opportunities for Opertations Research. European Journal of Operational Research, v. 101, n. 3, p. 419-429, 1997.

MARCHALEK, C. R. L. Estudo das lacunas de percepção no relacionamento empresa-terceirizado. Curitiba: PUCPR, 2005, 152 p. Dissertação (Mestrado). Programa de PósGraduação em Engenharia de Produção e Sistemas, Pontifícia Universidade Católica do Paraná, Curitiba, 2005.

MCIVOR, R. T.; HUMPHREYS, P. K.; MCAIEER, W. E. European car makers and their suppliers: changes at the interface. European Business Review, v. 98, n. 2, p. 87-99, 1998.

MENTZER, J. T. et al. Defining Supply Chain Management. Journal of Business Logistics, v. 22, n. 2, p. 1-25, 2001.

METCALF, L. E.; FREAR, C. R.; KRISHNAN, R. Buyer-seller relationships: An application of the IMP interaction model. European Journal of Marketing, v. 26, n. 2, p. 27-46, 1992.
MOHR, J.; SPEKMAN, R. Characteristics of partnership success: Partnership attributes, communication behavior, and conflict resolution techniques. Strategic Management Journal, v. 15, n. 2, p. 135152, 1994

MORGAN, R. M. HUNT S. D. The commitment-trust theory of relationship marketing. Journal of Marketing, v. 58, n. 3, p. 20-38, 1994

NOOTEBOOM, B. Inter-firm alliances: analysis and design. London: Routledge, 1999.

OLIVEIRA, F. C.; SANTOS, C. A. P. N. Terceirização no processo de desenvolvimento de sistemas de informações. In: XXIV ENCONTRO NACIONAL DA ASSOCIAÇÃO NACIONAL DOS PROGRAMAS DE PÓSGRADUAÇÃO EM ADMINISTRAÇÃO, 2000, Florianópolis. Anais... Florianópolis: ANPAD, 2000. 1 CD-ROM.

OLIVER, C. Determinants of interorganizational relationships: integration and future directions. Academy of Management Review, v. 15 , n. 2, p. 241-265, 1990.

PARTVATIYAR, A.; SHETH, J. N. The domain and conceptual foundations of relationship marketing. Handbook of Relationship Marketing. London: Ed. By Sheth, J. N. \& Parvatiyar, A., 2000.

PRADO, E. P.V.; TAKAOKA, H. Os fatores que motivam adoção da terceirização da tecnologia de informação: uma análise do setor industrial de São Paulo. In XXV ENCONTRO NACIONAL DA ASSOCIACÃO NACIONAL DOS PROGRAMAS DE PÓSGRADUACCÃO EM ADMINISTRACCÃO, 2001, Campinas. Anais...Campinas: Anpad, 2001. 1 CD-ROM. 


\section{- Referências}

PRAHALAD, C. K. ; HAMEL, G. The core competence of the corporation. Harvard Business Review, p. 3-15, maio/jun. 1990.

QUEIROZ, C. A. R. S. Manual de Terceirização: onde podemos errar no desenvolvimento e na implantação dos projetos e quais são os caminhos do sucesso. São Paulo: STS, 1998.

REZENDE, W. Terceirização: A Integração Acabou? Revista de Administração de Empresas - FGV/SP. São Paulo, v. 37, n. 4, p. 6-15, out./dez. 1997.

RING, P.S.; VAN DE VEN, A.H. Structuring cooperative relationships between organisations. Strategic Management Journal, v. 13 , p. $483-98,1992$.

RODRIGUES, A. M. Cluster e competitividade: um estudo da concentração de micro e pequenas empresas de alimento no município de Marilia/SP. São Carlos: EESC-USP, 2003
191 p. Tese (Doutorado). Programa de Pós-Graduação em Engenharia Mecânica, Escola de Engenharia de São Carlos, Universidade de São Paulo. São Carlos, 2003.

SINCLAIR, D.; HUNTER, L.; BEAUMONT, P. Models of customer-supplier relations. Journal of General Management, v. 22, n. 2, p. 56-75, 1996.

SKINNER, W. Manufacturing in the Corporate Strategy, New York: John Wiley, 1978.

SLACK, N. et al. Administração da Produção. São Paulo: Atlas, 1997.

SPEKMAN, R. E. Strategic Supplier Selection: Understanding Long-Term Buyer Relationships. Business Horizons, v. 31, n. 4, p. 75-81, 1988.
VILLA, F; PANIZZOLO, R. Buyer-subcontractor relationships in the Italian clothing industry. An interpretive framework. International Journal of Operations \& Production Management, v. 16, n. 7, p. 38-61, 1996.

WATSON, H.; RAINER, K.; HOUDESHEL, G. Executive Information Systems: emergence development impact. New York: John Wiley \& Sons, 1982

WILLIAMSON, O. E. The Economic Institutions of Capitalism: Firms, Markets, Relational Contracting. New York: The Free Press, 1985.

WILSON, D. T. An integrated model of buyer-seller relationships. Journal of the Academy of Marketing Science. v. 23, n. 4, p. 335-345, 1995.
WINTER, E. M. W. Introdução à Bioestatística. Curitiba: Departamento de Estatística, Universidade Federal do Paraná. 2002.

YAN, A.; GRAY, B. Bargaining power management control, and performance in United States-China joint ventures: a comparative case study. Academy of Management Journal, v. 37, n. 6, p. 14761517,1994

ZINELDIN, M; JOHANNISSON, B.; DANDRIDGE, T. Strategic Relationship Management: A Multi-dimensional Perspective. Alqmqvist \& Wilksell International, 1997.

ZINELDIN, M; JONSSON, P. An examination of the main factors affecting trust/ commitment in supplier-dealer relationships: empirical study of the Swedish wood industry. The TQM Magazine, v. 12 n. 4 , p. $245-265,2000$.

\section{- Sobre os autores}

\section{Cleverson Regis Lino Marchalek}

Pontifícia Universidade Católica do Paraná (PUCPR)

Programa de Pós-Graduação em Engenharia de Produção e Sistemas (PPGEPS)

Aluno de mestrado

End.: Rua Imaculada Conceição, 1155 - Curitiba - PR - CEP 80215-901

Tel.: (41) 3271-1344

E-mail: marchalek@yahoo.com

Site: www.produtronica.pucpr.br

\section{Marcelo Giroto Rebelato}

Pontifícia Universidade Católica do Paraná (PUCPR)

Programa de Pós-Graduação em Engenharia de Produção e Sistemas (PPGEPS)

Professor

End.: Rua Imaculada Conceição, 1155 - Curitiba - PR - CEP 80215-901

Tel.: (41) 3271-1344 / 3271-1332

E-mail:m.giroto@pucpr.br

Site: www.produtronica.pucpr.br

\section{Isabel Cristina Rodrigues}

Faculdade de Administração e Negócios de Monte Alto

Professora

End.: Rua Wady Elias, 191 - CEP 15910-000 - Monte Alto - SP

Tel.: (16) 3242-1742

E-mail: isabelrodrigues2@yahoo.com.br 
APÊNDICE A - Questionário aplicado às empresas contratantes

\section{QUESTÕES}

Baseado em suas experiências passadas e presentes, qual a importância da comunicação para o relacionamento de trabalho com as empresas terceirizadas?

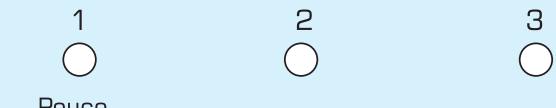
importante $\stackrel{4}{\circ} \stackrel{4}{0}^{3}$ $\stackrel{5}{\bigcirc}$
6

7



Muito importante

Baseado em suas experiências passadas e presentes, como você caracteriza o nível de confiança que sua empresa possui no relacionamento de trabalho com o terceirizado?
1
2
4
0
$\bigcirc$
O
0
$\stackrel{5}{0} \bigcirc^{6}$
$\stackrel{5}{\bigcirc^{\circ}}$
Pouco
importante
O
Muito
importante

Minha empresa acredita que a cooperação é fundamental para o relacionamento como o terceirizado.
1
2

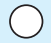
0
0
0
$\stackrel{5}{\bigcirc}$
${ }^{6}$
7
0
Discorda
fortemente

Minha empresa acredita que estar comprometido com o terceirizado é fundamental para o relacionamento.

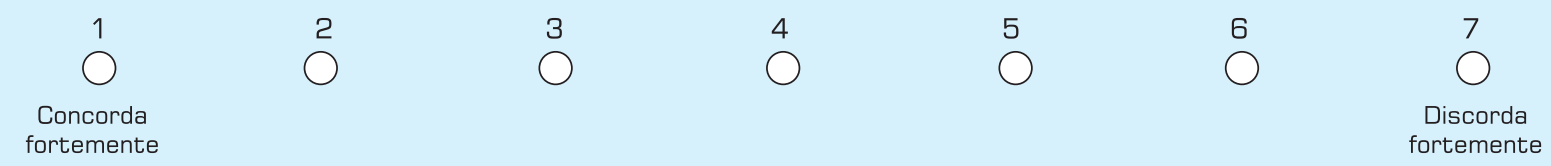

Os riscos e as recompensas devem ser igualmente divididos entre nossa empresa e o terceirizado.

1

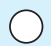

Concorda

fortemente

\section{2}

0
3

0
4

0
5

0
6

○
7 
APÊNDICE B — Questionário aplicado aos terceirizados



